\title{
Development of an HTML5 Library for Building SCORM Learning Objects
}

\author{
Adelfo Salazar-Salazar, Carlos Felipe García-Hernández \\ National Institute of Electricity and Clean Energies, Cuernavaca, Morelos, \\ Mexico \\ adelfosalazar@gmail.com, cfgarcia@ineel.mx
}

\begin{abstract}
This work presents the development of an HTML5 library that allows the building of LO's (Learning Objects) compatible with the SCORM (Shared Content Object Reference Model) standard and the way in which they have been implemented within the "Learning Management, Capture of Experience and Content Repository" (GACER, for its acronym in Spanish) learning platform from the National Institute of Electricity and Clean Energies (INEEL, for its acronym in Spanish). It describes the way in which they are usually developed, as well as the main advantages of using this new tool as to improve the contents interactivity level through the integration of $3 \mathrm{D}$ animations and their accessibility from mobile devices.
\end{abstract}

Keywords: SCORM, HTML5, LMS, e-Learning, m-Learning, learning objects.

\section{Introduction}

Many institutions confront different situations that induce them to use training systems. The professional updating and the rotation of staff are some of them, being very important the recovery of the workers' knowledge to be able to retransmit it. There are several ways to accomplish this task and e-learning is one of them. With the objective of offering a quality product within this modality, INEEL uses the SCORM standard, which according to the definition provided by the author in [1] is " a set of technical specifications in learning area through the Internet (e-learning) that define the structure of the contents; their behavior and the behavior of the LMS when it comes to hosting and executing said contents".

The way in which this learning material has been developed, until recently, completely fulfilled the requirements of the projects. However, the current trends, the breakdown of some technologies and the requirements of the projects have resulted in this type of material not satisfying to the best degree the clients expectations, mainly due to the low level of interactivity and personalization of the learning material. 


\section{Related Work}

In [2], authors explain in detail the way to achieve SCORM activities configuration, involving sub-activities or several SCO (as named in SCORM standard, being learning objects containing teaching materials), following the guidelines proposed by ADL with Template 10 and Educative Model 3.

In [3], authors explain an application of pedagogical tools to an on-line course for elearning, including an example of a course specification showing an activity, an example of a script for an activity, and a course development model.

\section{Analysis of the Problem}

Content development is usually done using authoring tools such as Adobe Captivate, and the main disadvantage of working in this way is that the Flash format (file format included in SCORM packages) is no longer supported by systems in current mobile devices such as Android and iOS, because manufacturers of this systems have determined that their use poses a risk to user information and impairment of device performance. Because of this, we have chosen to use HTML5 for the development of the tool which completely solves this problem.

For issues related to professional profiles that can participate in content development and game development work, there are various opinions on which of both alternatives is best. But opinions agree when talking about the advantages that comes with using a mobile device to present this material including playback and video. In [4] the author mentions "HTML5 is very well supported on mobile devices, and on iOS devices CSS3 $3 D$ transitions even hardware accelerated. The video is fully compatible with native (often hardware-based) H.264 capabilities for web application and video playback". Table 1 shows some of the main advantages HTML5 has over Flash technology.

Table 1. Comparison of Flash and HTML5 technologies

\begin{tabular}{lcc}
\hline \multicolumn{1}{c}{ Characteristics } & \multicolumn{2}{c}{ Technologies } \\
& Flash & HTML5 \\
\hline Compatibility with mobile devices & NO & YES \\
Independence of plugins for execution & NO & YES \\
Prescription of proprietary software for use & NO & YES \\
Compatibility with current web browsers & NO & YES \\
Life expectations & NO & YES \\
\hline
\end{tabular}

The author also mentions that there are already authoring tools that allow export to HTML5. However, even using some of them, there are still a number of disadvantages, which will vary according to the software used, of course, the degree of customization that is desired to reach the product and the requirements of the project. 


\section{$4 \quad$ Main Drawbacks of Some Authoring Tools}

In the first instance, it is important to recognize that this type of tools offer many advantages, mainly that for the elaboration of contents with a low level of interactivity can be dispensed, in many cases, of a software specialist. However, there are a number of drawbacks described below:

- Non-admission of 3D animations.

It is not possible to include 3D animations in LO's and with detection of actions on the animation. In addition, the material must be considered capable of running on mobile devices without requiring special hardware.

- Incompatibility with some web browsers.

The material requires the use of a content player, which is not compatible with some web browsers such as Internet Explorer and Firefox.

- Software license payment.

These tools, for the most part, are proprietary software, so they require the purchase of a license, which is usually expensive.

- Dependency of a company that owns.

The user is limited to the functions that integrate these tools at the time of purchase. To access new features will be subject to the appearance of an update, which can take a long period of time.

Some of these tools have the ability to include scripts to control the SCORM-LMS interaction, however, it is still difficult to apply new technologies and functionalities without resorting to source code modification, which is complicated to alter when they have already been applied techniques such as code obfuscation or minification.

\section{$5 \quad$ Technological Solution and First Results}

An HTML5 library has been developed that allows the elaboration of interactive LO's compatible with SCORM and that solves the problems listed in the previous section. The main technical details of development are shown below:

- Type of tool: Web, HTML5 library.

- Content definition language (markup language): HTML5.

- Programming language: JavaScript.

- Metadata description language: XML.

- Learning Objects Standard: SCORM.

- Design regulations (visual appearance): Material Design.

- Compatibility: With current web browsers (including mobile, obviously).

The library has the following features: 
- Generation of views and inclusion of navigation controls: Integration of controls that facilitate navigation between different views is allowed. These features allow you to show the content in a better organized way.

- Insertion of multimedia content with scalable design: Insertion of multimedia elements, including 2D and 3D animations, to which scalability functions are applied to preserve a persistent appearance between different screen sizes.

Attractive visual design of components: The design rule Material Design has been applied, which gives a more attractive and modern appearance to the components used within the LO's.

- Easy integration of 2D animations: We worked on the inclusion of functions that allow the loading of 2D animations in a simple way, non-simultaneous execution with other animations to avoid slowing, etc.

- Easy integration and correct operation of 3D models: In this activity, a basic script was developed for the implementation of 3D animations in a web using HTML5 and JavaScript, through the library Three.js. An example of this implementation can be seen in Fig. 1.

The script involves, among other things, the following functionalities:

- Adding interactive properties to each of the 3D elements, such as zoom, rotation, and navigation in the scenarios.

- Configuring events to detect user interactions.

- Inclusion of interactive learning activities.

They are included were interactive activities are, to contribute to user participation during the learning process; the puzzle is an example.

- Template for the preparation of evaluation activities.

Templates were integrated to allow evaluation. An example is the exams with questions of type True / False, multiple choice, etc.

- Monitoring user activity and interacting with the LMS using CMI tracking elements.

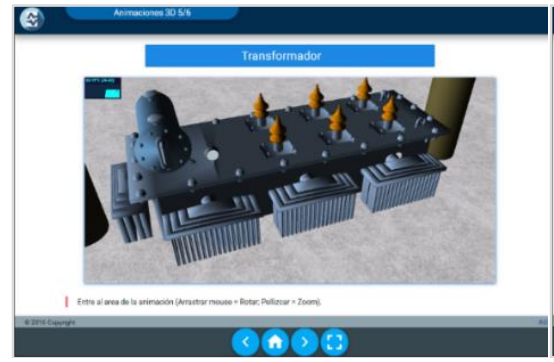

Fig. 1. 3D Animation inserted in an SCO

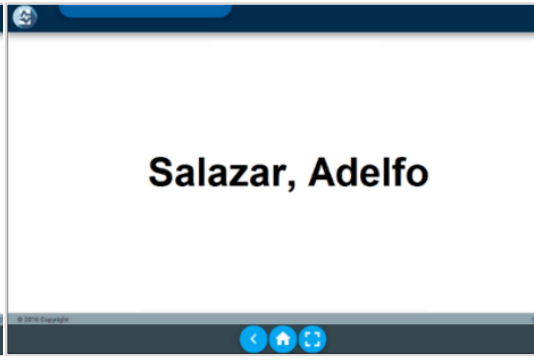

Fig. 2. Obtaining the value of the cmi.core.student_name.

It was possible to establish communication between the LO's and the LMS platform by developing the drivers "cmi_controller1.2.js" and "cmi_controller2004.js" for versions 1.2 and 2004 respectively.

Some of the CMIs with which it is possible to interact are: 
- cmi.core.student_id: Identifies the connected student.

- cmi.core.student_name: Name of connected student (Fig. 2).

- cmi.core.score.raw (RW): Qualification of the evaluation activity.

- cmi.core.score.min (RW): Minimum assignable score.

- cmi.core.score.max (RW): Maximum assignable score.

- cmi.core.lesson_status: Indicates if the student has finished and satisfies the conditions of the SCO.

- cmi.core.exit: Indicate how or why the student left the SCO. Allows the completion of the activity.

\section{SCORM-based LO's Development Methodology}

Next, the roles and work methodology, represented in Fig 3, are described, which are typically followed in INEEL for the elaboration of the SCORM contents (in [5], an extended and detailed description of this methodology is presented).

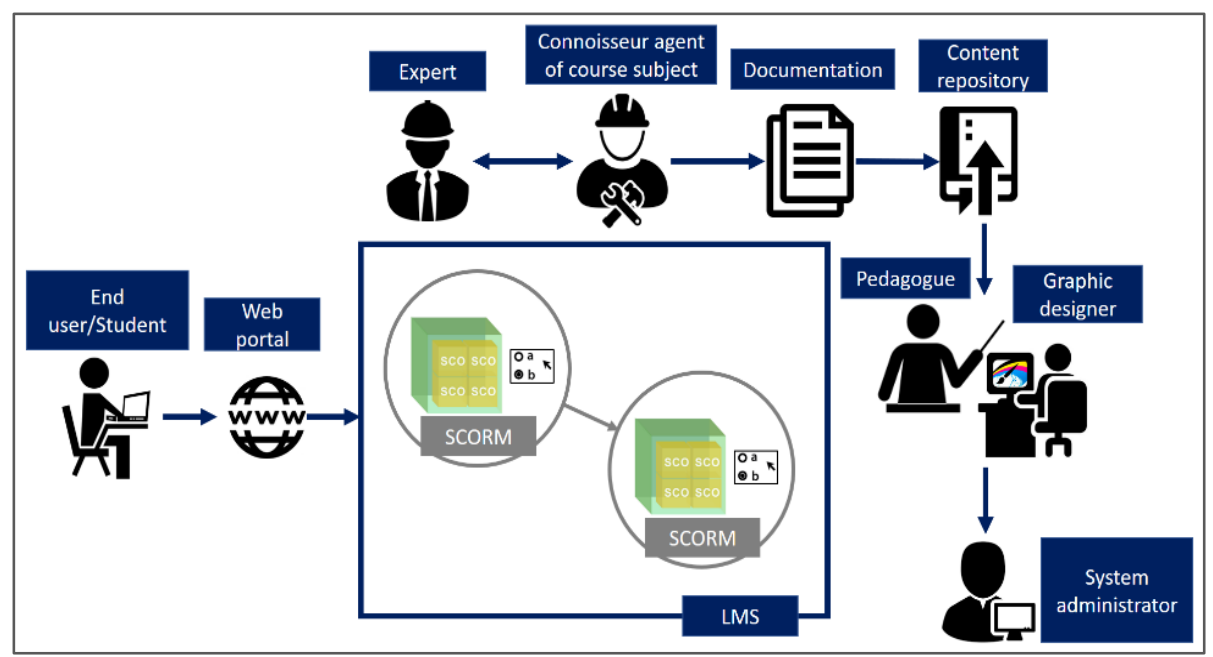

Fig. 3. SCORM content development methodology.

- Expert: Person, in the team, with extensive experience and the highest degree of knowledge about the subject of the LO's, generally, part of the client team.

- Connoisseur agent of course subject: Person with domain of the course subject and responsible for establishing direct communication with the expert to be able to relay the information provided.

- Documentation and content repository: The agent explicitly documents the information provided by the expert and stores it in a content repository.

- Pedagogue and graphic designer: The pedagogue analyzes the documented information and determines the way in which it will be presented to facilitate learning of users. Graphic designer elaborates the multimedia contents. 
- System administrator: Person responsible for preparing the training environment and preparing the LO's based on the information provided by pedagogue and designer.

- End user/Student: Person who accesses the web portal and consumes course information.

\section{$7 \quad$ Results}

This tool allowed the elaboration of the first content corresponding to the Smart Grid course integrated to the INEEL GACER platform. Currently this material is available in the Smart Grid Laboratory and it is used to promote the capabilities of the Advanced Training Systems and Simulation Department (GSACyS, for its acronym in Spanish) from INEEL.

Specifically speaking about the tool, a basic development was established for the integration of practically any resource within the LO's, given the source code disposition and access to all the main functionalities developed for the composition of the library. It is mention also that to use this tool it is enough to have basic knowledge of HTML5 because, as mentioned before, the described functionalities have already been programmed and the content developer will only need to make the layout or definition of tags according to the development process that is described in a respective SCORM content development manual included with this tool.

\section{Future Work}

It has been considered mainly to continue the development and refinement of some features that already integrate the library, mainly regarding the inclusion of a greater amount of interactive activities of learning and evaluation, the manipulation of all the elements CMI in the versions 1.2 and 2004 of the standard SCORM, as well as the maximum simplification of the use of the tool, since in spite of already being able to elaborate the contents only by the definition of the document HTML5, it still can facilitate and improve the learning curve on the use of the tool.

\section{Conclusion}

This development allowed to break with some paradigms of work and give solution to the new formation needs, as well as to satisfy the change in the habits of consumption of information and the levels of interaction demanded presently, which allows to continue like distinguished producers of systems for online training and courses and to make contributions of greater satisfaction to customers' demands. This solution brings us one step closer to automating online courses delivered through an LMS, where the implementation of cmi tracking elements is the last step of the intelligent tutoring. 


\section{References}

1. Laguna, M.: Introduction to the SCORM Reference Model. Zaragoza University, Spain (2011)

2. García, C. F., Sánchez, M., Jiménez, F. F.: Configuration of Template 10 and Educational Model 3 of ADL. Cuernavaca Morelos, Mexico (2013)

3. Ahmad, A., Ahmad, M., Marwa, A., Tamer, M., Ahmed, H., James, E., Kutluk, Ö.: Applying Pedagogical Concepts in Online Course Development. Mediterranean Virtual University (2005)

4. Jeremy, C.: How does HTML5 compare with Adobe Flash? California, United States (2011), https://www.quora.com/How-does-HTML5-compare-with-Adobe-Flash

5. Rodríguez, G., Molina, R., García, C. F., Mendoza, M. J.: Methodology for the Implementation of Distance Training. Cuernavaca Morelos, Mexico (2015) 